\title{
Programa "The pleasure of reading with pictures" a través de los textos iconográficos para el desarrollo de la comprensión de textos en inglés en estudiantes del nivel primario.
}

\section{Program "The pleasure of reading with pictures" through iconographic texts for the development of reading comprehension in English with the students of primary level.}

\author{
Huashuayo Carrasco, Esmeralda \\ EP Educación, Facultad de Ciencias Humanas y Educación, Universidad Peruana Unión
}

\begin{abstract}
Resumen
El objetivo de la presente investigación fue determinar la eficacia del programa "The pleasure of reading with pictures" a través de textos iconográficos para el desarrollo de la comprensión de textos en inglés como segunda lengua en los estudiantes del $5^{\circ}$ grado de primaria de la Institución Educativa Adventista "Portales del Saber", Vitarte-2014. El diseño de la investigación fue pre-experimental con enfoque cuantitativo. La muestra estuvo conformada por 19 estudiantes. Para el recojo de la información concerniente a la comprensión de textos en inglés se utilizó el instrumento "Guía de Observación" el que se utilizó para medir el nivel de logro tanto para la pre prueba como la post prueba. Para el proceso de análisis estadístico se utilizó el programa Statistical Package for Social Sciences (SPSS), versión 20.0. A través del estadígrafo T de student, en el pre test se obtuvo 63,2\% en el nivel deficiente y $36,8 \%$ en el nivel regular. En el post test, se obtuvo en el nivel deficiente $0 \%$, y en el nivel regular también se obtuvo el $0 \%$, es decir; todos los estudiantes alcanzaron un $100 \%$ en el nivel eficiente. El valor de significancia de 0,000 que se obtuvo en el post test significa según la regla de decisión que este valor es menor que el margen de error de 0.05 mostrando estadísticamente que el programa "The pleasure of reading with pictures" tuvo efectividad significativa para desarrollar la comprensión de textos en inglés. Estos resultados mostraron que los estudiantes mejoraron notablemente, ya que en un inicio alcanzaban un nivel deficiente y regular; pero, después de la ejecución del programa, todos los participantes lograron un nivel bueno.
\end{abstract}

Palabras clave: Comprensión lectora, textos iconográficos, actividades lúdicas, nivel literal, nivel inferencial.

\section{Abstract}

The aim of this research was to determine the effectiveness of the program "The pleasure of reading with pictures" through iconographic texts for the development of reading comprehension in English as a second language with the students of the $5^{\text {th }}$ grade of primary level from the Adventist Educational Institution "Portales del Saber", Vitarte-2014. The research design was preexperimental (quantitative approach) The sample was composed by 19 students. For information gathering concerning reading comprehension, an "Observation guide" was used to measure student's level of achievement. The software Statistical Package for Social Science (SPSS), version 20.0 was used for the statistical analyses. Through the T-student test we got $63,2 \%$ in low level and 36,8 in regular level in pretest. In the post test, we got $0 \%$ in the low level, and $0 \%$ in the regular level, it means that all students got $100 \%$ in the good level We got 0.000 in the post test as significance value that means according to the decision ruler that this value is lower than the error range of 0.05 showing statistically that the program "The pleasure of reading with pictures" had significant effectiveness to develop the skills of reading comprehension in English. The results showed that students improved notably since at the beginning students showed a low and regular level, but after the execution of the program, all the participants got a good level.

Key words: Reading comprehension, iconographic texts, playful activities, literal level, and inferential level.

\section{Introducción}

Los resultados de pruebas tanto nacionales como internacionales han evidenciado la gran dificultad que tienen nuestros estudiantes cuando se enfrentan a un texto escrito. El nivel de comprensión lectora es muy bajo, incluso las preguntas que deben responder en los exámenes son mal interpretadas, en muchas ocasiones, por los estudiantes. Si este problema ocurre en su propio idioma, aún más compleja será en el aprendizaje y la comprensión de textos de una lengua extranjera, en este caso, el inglés.

La idea de investigación del presente estudio parte de uno de los problemas preocupantes en educación, después de los resultados alarmantes de la Evaluación Internacional de Estudiantes (Programme for International Student Assessment: PISA) del año 2009 y 2012 y pruebas nacionales, lo cual evidencian que la comprensión de textos tanto en inglés como en español son deficientes. A nivel nacional, teniendo en cuenta el análisis de los resultados de las pruebas del año 2011, en el componente de 
inglés, se pudo hallar que, 22 de cada 100 estudiantes alcanzaron los niveles B1 o B+, que corresponden a aquellos que logran comunicarse efectivamente. El 17\% se ubicó en el nivel A2: comprenden frases y 22 expresiones de uso frecuente relacionadas con su entorno cercano y se comunican para realizar tareas simples y cotidianas. El 32\% quedó en el nivel A1, lo que quiere decir que comprenden y utilizan frases sencillas para relacionarse de forma elemental. El 29\% restante no alcanzó el nivel A1. Se puede inferir que la mayoría de los estudiantes se encuentran en el nivel A1 (PISA, 2009). Para que esta problemática no siga su curso, es necesario empezar desde temprana edad, es decir, en la etapa donde el estudiante inicie la lectura en su propia lengua.

La presente investigación plantea la necesidad de desarrollar una práctica transformadora de la enseñanza y aprendizaje del inglés con el propósito de obtener una mejor comprensión lectora en una segunda lengua. La transformación de la enseñanza y el aprendizaje de la lectura se fundamenta en los paradigmas del aprendizaje significativo Ausubel citado por Viera (2003) y cooperativo sustentados por las teorías socioconstructivistas donde los estudiantes puedan crear, mejorar, negociar significados e interactuar en una lengua extranjera. A pesar que el inglés forma parte del currículo nacional, existen pocas investigaciones en el área de inglés para mejorar la comprensión lectora en estudiantes de primaria. De allí, la necesidad de esta investigación de mejorar los procesos de enseñanza para mejorar la comprensión lectora en una segunda lengua en estudiantes del V ciclo de la Educación Básica Regular.

Esta investigación constituye un aporte en el campo educativo puesto que la planificación de estrategias didácticas lúdicas en el área de inglés facilitará el aprendizaje del idioma inglés. Asimismo, desarrollará una mejor comprensión de textos del mismo, de una forma grata y amena. De la misma manera, le permite al docente ser más que un simple intermediario entre el currículo establecido y los alumnos. Las actividades lúdicas se convierten en estrategias de aprendizaje con una escala de alto grado, porque lo que los alumnos aprenden jugando, difícilmente se les olvida.

Todo lo antes descrito dio motivo al objetivo de realizar la presente investigación lo cual consiste en determinar la eficacia del programa "The pleasure of reading with pictures" a través de los textos iconográficos que consta de actividades lúdicas con el uso de imágenes para la mejor comprensión de textos en inglés y las estrategias que se dan en los tres momentos de la lectura, antes, durante y el después, bajo las capacidades de comprensión lectora propuesta por Anderson (2001) adecuadas en la concepción literaria de PISA asumidas en el área de Comprensión lectora por la Unidad de Medición de la Calidad (UMC) del Ministerio de Educación. (2013).

\section{Método}

Según su finalidad, este estudio es de tipo aplicada pues depende de los descubrimientos y avances de la investigación básica y ser enriquece con ellos, pero se caracteriza por su interés en la aplicación, utilización y consecuencias prácticas de los conocimientos. Además, busca en el estudiante el conocer para hacer, para actuar, para construir, para modificar. El estudio es de enfoque cuantitativo, porque tiene características de medición para su respectiva recolección de datos y análisis estadístico mediante el instrumento de evaluación "Guía de observación" que permitió evaluar el proceso de aprendizajes y en base de los resultados obtenidos se aplicó el programa "The pleasure of reading with pictures" permitiendo fundamentar la existencia de la eficacia del programa para responder a las hipótesis determinadas de la presente investigación. La población estuvo constituida por 19 estudiantes del $5^{\circ}$ grado único del nivel primario de la Institución Educativa Particular Adventista "Portales del Saber", de los cuales el $57.9 \%$ eran de género masculino y el $42.1 \%$ de género femenino. Un $89.5 \%$ de los estudiantes se encontraban viviendo en material noble, un $5.3 \%$ en material de adobe y en otros en un $5.3 \%$.

Para la recolección de información se utilizó dos instrumentos: una guía de observación y una lista de cotejo (que sirvió de apoyo a la guía, conteniendo los mismos ítems de evaluación) que fue validado por el juicio de 5 expertos y por el coeficiente de V-Aiken. Para medir la variable de comprensión de textos en inglés, la Guía de observación, constó de 3 partes los cuales fueron las instrucciones, datos generales y 2 dimensiones para la evaluación de los datos del instrumento que fueron: Dimensión 1, comprensión en nivel literal con 6 ítems, y dimensión 2, comprensión en nivel inferencial con 5 ítems, de acuerdo con la operacionalización de las variables y con los niveles de evaluación, deficiente, regular y bueno. El nivel de confiabilidad del instrumento $(0,822)$ se midió en base a la medida de consistencia de Alfa de Crombach (Valores para la interpretación del coeficiente alpha).

El programa de investigación se llevó a cabo dentro de un módulo titulado: "Talking about past activities", donde todas las lecturas estaban redactadas en pasado, dentro de contextos reales de los Estados Unidos en su mayoría. Todo el vocabulario que contenía cada lectura se presentaba en forma gradual a través de actividades lúdicas. La técnica de evaluación que fue utilizada por la investigadora en cada sesión del módulo fue la observación sistemática, la cual se valió de una lista de cotejo (que contenía los mismos indicadores de la Guía de observación) Por ser la muestra menor de 30 se utilizó la prueba de Bondad de Ajuste a la Curva Normalidad de Shapiro - Wilk, y para el análisis estadístico, por ser de datos paramétricos en el pre y post test (resultando un 0,617 y 0,062 mayores que 0,05) se aplicó el estadístico de prueba T de student. Todo el cálculo estadístico se dió a través del software Statistical Package for the Social Sciences (SPSS), versión 20.0. Los análisis se realizaron con un 95\% de confianza y un 5\% de significancia de error. 


\section{Resultados y Discusión}

Los resultados de los análisis descriptivos relevantes para las investigaciones se muestran en la tabla 1 donde indica que el 63.2\% de los estudiantes tuvieron un nivel de comprensión de textos en inglés "Deficiente" en el pre test, lo que significa que los estudiantes fueron capaces solo de identificar información explícita del texto o inferir alguna información con ayuda del texto. Como se observa, este porcentaje disminuyó a un $0.0 \%$ en el post test (ningún participante ha obtenido el nivel deficiente). En el pre test, el 36.8\% de los estudiantes obtuvieron un nivel de comprensión de textos en inglés "Regular" lo que significa que supieron reconocer los personajes principales del texto, reconocer lugares y escenarios del texto, identificar el tiempo en que sucedieron los hechos, identificar palabras y frases propuestas que completen el sentido del enunciado y deducir el significado de las palabras mediante imágenes según el contexto. En el post test, el 100.0\% obtuvo el nivel "Eficiente", lo que significa que recuperaron información de los diversos textos escritos, reorganizaron información de los diversos textos escritos. Asimismo, infirieron el significado de los diversos textos escritos.

Tabla 1 Nivel de Comprensión de textos en inglés de los estudiantes del quinto grado de primaria de la I.E.A.

"Portales del Saber"

\begin{tabular}{lcccc}
\hline \multirow{2}{*}{ Niveles } & \multicolumn{2}{c}{ Antes } & & \multicolumn{2}{c}{ Después } \\
& Frecuencia & Porcentaje & Frecuencia & Porcentaje \\
\hline Deficiente & 12 & $63,2 \%$ & 0 & $0.0 \%$ \\
Regular & 7 & $36.8 \%$ & 0 & $0.0 \%$ \\
Eficiente & 0 & $0,0 \%$ & 19 & $100.0 \%$ \\
Total & $\mathbf{1 9}$ & $\mathbf{1 0 0 , 0 \%}$ & $\mathbf{1 9}$ & $\mathbf{1 0 0 , 0 \%}$ \\
\hline
\end{tabular}

Respecto al nivel de comprensión literal los resultados de la tabla 2 indican que los estudiantes obtuvieron un $57.9 \%$ en el pre test en el nivel "Deficiente" lo que significa que los estudiantes sólo pudieron reconocer los personajes principales del texto, reconocer lugares y escenarios del texto. En el post test, ningún participante obtuvo el nivel deficiente. En el pre test, el $42.1 \%$ obtuvo el nivel "Regular" lo que significa que los estudiantes además de reconocer los personajes principales del texto y reconocer lugares y escenarios del texto, identificaron palabras y frases propuestas del texto que completen el sentido del enunciado. Este nivel tuvo una mejora enorme ya que en el pos test ningún participante obtuvo el nivel regular. Por último, en el pos test, el 100.0\% de los participantes alcanzó el nivel "Eficiente" lo que significa que los estudiantes pudieron recuperar información explícita en los textos en inglés (personajes principales, tiempo, lugares) y reorganizar información de los mismos (reconstruir la secuencia del texto e identificar las palabras y frases propuestas que completen el sentido del enunciado).

Tabla 2

Nivel de Comprensión literal de los estudiantes del quinto grado de primaria de la I.E.A. "Portales del Saber"

Antes

Niveles Frecuencia

Porcentaje

$57.9 \%$

$42.1 \%$

$0.0 \%$

$100,0 \%$

\section{Después}

Frecuencia Porcentaje

$0 \quad 0.0 \%$

0

$0.0 \%$

19

$100.0 \%$

19

$100,0 \%$

En comprensión inferencial, los resultados que muestra la tabla 3, declara que el $78.9 \%$ de los estudiantes tiene un nivel "Deficiente" en el pre test, lo que indica que la mayoría de ellos tiene dificultades para acercarse al texto y deducir datos implícitos del mismo con ciertas limitaciones. Aún pueden deducir el tipo de texto. Tienen dificultad para deducir la idea y el tema del texto. En el post test, ningún participante obtuvo el nivel deficiente. El 21.1\% de los estudiantes alcanzaron un nivel "Regular" en el pre test, es decir que además de inducir el tipo de texto, pueden inferir palabras con el posible significado e inferir el significado de las palabras mediante gráficos e imágenes según el contenido. En el post test, ningún estudiante obtuvo el nivel regular. En el pos test, el 100.0\% de los mismos alcanzó al nivel "Bueno", lo que significa que además de deducir el tipo de texto, inferir palabras con el 
posible significado, inferir el significado de las palabras mediante gráficos e imágenes según su contenido, también logran deducir el tema y la idea principal del texto.

Tabla 3

Nivel de Comprensión inferencial de los estudiantes del quinto grado de primaria de la I.E.A. "Portales del Saber"

\begin{tabular}{lcccc}
\hline \multicolumn{2}{c}{ Antes } & \multicolumn{2}{c}{ Después } \\
\hline Niveles & Frecuencia & Porcentaje & Frecuencia & Porcentaje \\
Deficiente & 15 & $78.9 \%$ & 0 & $0.0 \%$ \\
Regular & 4 & $21.1 \%$ & 0 & $0.0 \%$ \\
Eficiente & 0 & $0.0 \%$ & 19 & $100.0 \%$ \\
Total & $\mathbf{1 9}$ & $\mathbf{1 0 0 , 0 \%}$ & $\mathbf{1 9}$ & $\mathbf{1 0 0 , 0 \%}$ \\
\hline
\end{tabular}

La tabla 4 muestra la comparación de los resultados según la variable dependiente "Comprensión de textos en inglés" donde podemos observar que un $36.8 \%$ del género masculino se encontró en el nivel "Deficiente" en el pre test, en cambio para el pos test se superó ya que no se encontró a ningún niño en el nivel deficiente. El 21.1\% de este género se ubicó en el nivel "Regular", lo cual también fue superado en el post test porque no se encontró a ningún niño en este nivel. Por el contrario, en el post test, los niños alcanzaron un $57.9 \%$ de nivel "Bueno" lo cual representa su totalidad. En el género femenino, podemos observar que el $26.3 \%$ alcanzaron un nivel "Deficiente" en el pre test, lo cual fue superada en el post test porque no se encontró a ninguna niña en este nivel. El 15.8\% de las niñas alcanzaron el nivel "Regular" en el pre test, lo cual fue superada ya que en el post test no se halló a ninguna niña en este nivel. Por último, el $42.1 \%$ de este género alcanzó el nivel "Eficiente", lo cual representa a su totalidad. En conclusión, podemos aseverar que los niños y las niñas obtuvieron resultados satisfactorios en el pos test.

Tabla 4

Análisis comparativo del género según la variable "Comprensión de textos en inglés”

\begin{tabular}{lcccccccc}
\hline \multirow{2}{*}{ Género } & \multicolumn{3}{c}{ Antes } & & & \multicolumn{3}{c}{ Después } \\
& Deficiente & Regular & Eficiente & Total & Deficiente & Regular & Eficiente & Total \\
\hline Masculino & $36.8 \%$ & $21.1 \%$ & $0,00 \%$ & $57.9 \%$ & $0.0 \%$ & $0.0 \%$ & $57.9 \%$ & $57.9 \%$ \\
Femenino & $26.3 \%$ & $15.8 \%$ & $0,00 \%$ & $42.1 \%$ & $0.0 \%$ & $0.0 \%$ & $42.1 \%$ & $42.1 \%$ \\
Total & $63.2 \%$ & $36.8 \%$ & $0,00 \%$ & $100,0 \%$ & $0.0 \%$ & $0.0 \%$ & $100.0 \%$ & $100,0 \%$ \\
\hline
\end{tabular}

Para realizar la prueba de hipótesis de la variable dependiente: comprensión de textos en inglés se formularon la hipótesis nula y alterna las cuales para la hipótesis general proponen que, Ho: La aplicación del programa "The pleasure of reading with pictures" no tiene efectividad significativa para el desarrollo de las capacidades de comprensión de textos en inglés en los estudiantes del $5^{\circ}$ grado de primaria de la Institución Educativa Adventista "Portales del Saber" en Ate-Vitarte, Ha: La aplicación del programa "The pleasure of reading with pictures" tiene efectividad significativa para el desarrollo de comprensión de textos en inglés en los estudiantes del $5^{\circ}$ grado de primaria de la Institución Educativa Adventista "Portales del Saber" en Ate-Vitarte. Y según la regla de decisión: se acepta Ho si $\mathrm{p}>/=\alpha$, de lo contrario se rechaza si $\mathrm{p}<\alpha$ siendo que $\alpha$ es igual 0,05 . Y como ya se mencionó previamente se utilizó el estadístico de prueba paramétrico de T de Student mediante el cálculo estadístico. 


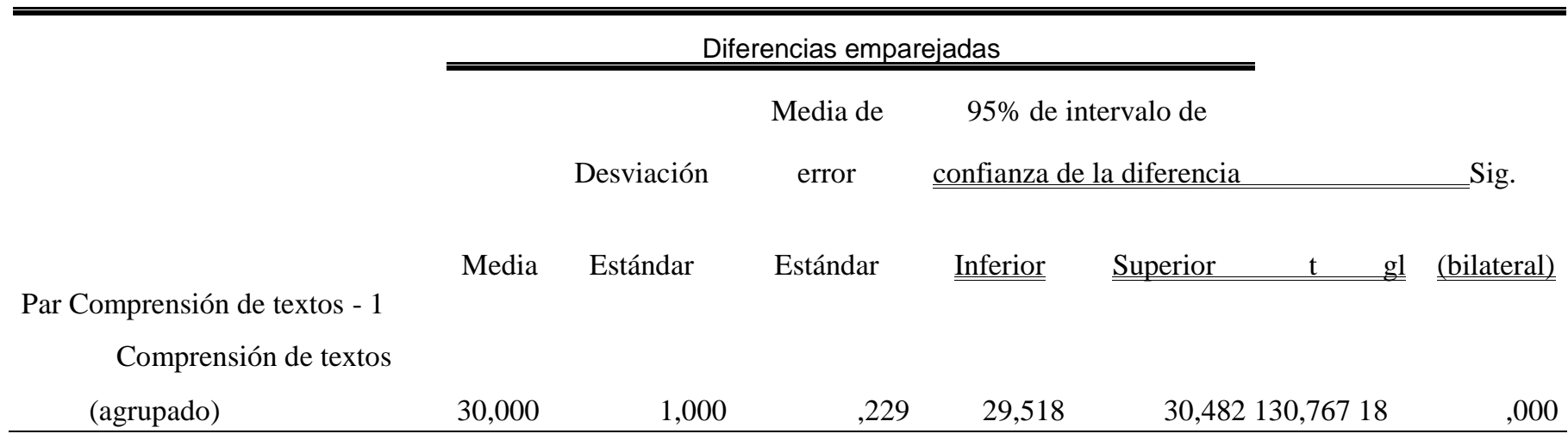

Por los datos obtenidos en la tabla 5 observamos que $\boldsymbol{p}<\mathbf{0 , 0 5}$ (significancia bilateral ,000) de acuerdo con la regla de decisión, por ello podemos afirmar que existe evidencia suficiente para rechazar la hipótesis nula; puesto que se acepta la hipótesis alterna: la aplicación del programa "The pleasure of reading with pictures" tiene efectividad significativa en el desarrollo de la comprensión de textos en inglés en los estudiantes del nivel primario de la Institución Educativa Particular Adventista "Portales del Saber" en Ate-Vitarte.

La prueba de hipótesis específica 1 en base a la dimensión, Comprensión en nivel literal, formulan que Ho: La aplicación del programa "The pleasure of reading with pictures" no tiene efectividad significativa en el desarrollo de la compresión de textos en inglés en los estudiantes del $5^{\circ}$ grado del nivel primario de la Institución Educativa Particular Adventista "Portales del Saber" en Ate-Vitarte. Ha: La aplicación del programa "The pleasure of reading with pictures" tiene efectividad significativa en el desarrollo de la compresión de textos en inglés en los estudiantes del $5^{\circ}$ grado del nivel primario de la Institución Educativa Particular Adventista "Portales del Saber" en Ate-Vitarte. Se aplicó el estadístico de prueba T de student por tratarse de datos paramétricos como ya se explicó previamente.

Tabla 6

T de Student para la dimensión comprensión en nivel literal

\section{Diferencias emparejadas}

95\% de intervalo de Media de

confianza de la

Desviación

error

diferencia

Sig.

\begin{tabular}{|c|c|c|c|c|c|c|c|c|}
\hline \multirow{2}{*}{\multicolumn{2}{|c|}{$\underline{\text { Media }}$}} & \multirow[b]{2}{*}{$\underline{\text { estándar }}$} & \multirow[b]{2}{*}{ estándar } & & & \multirow[b]{2}{*}{$\mathrm{gl}$} & \multirow[b]{2}{*}{$\underline{\text { (bilateral) }}$} \\
\hline & & & & $\underline{\text { Inferior }}$ & $\underline{\text { Superior }}$ & $\underline{\mathrm{t}}$ & & \\
\hline Par 1 Literal - Literal & $-7,579$ & 1,216 & ,279 & $-8,165$ & $-6,993$ & $-27,160$ & 18 & 000 \\
\hline
\end{tabular}

Por los datos obtenidos en la tabla 6 observamos que $\boldsymbol{p}<\mathbf{0 , 0 5}$ (significancia bilateral,000) de acuerdo con la regla de decisión, por ello podemos afirmar que existe evidencia suficiente para rechazar la hipótesis nula según la regla de decisión; puesto que se acepta la hipótesis alterna: la aplicación del programa "The pleasure of reading with pictures" tiene efectividad significativa en el desarrollo de la comprensión de textos en inglés en el nivel literal, en los estudiantes del $5^{\circ}$ grado del nivel primario de la Institución Educativa Particular Adventista "Portales del Saber" en Ate-Vitarte.

La prueba de hipótesis específica 2 en base a la dimensión, Comprensión en nivel inferencial, formulan que Ho: La aplicación del programa "The pleasure of reading with pictures" no tiene efectividad significativa en el desarrollo de la compresión de textos en inglés en nivel inferencial en los estudiantes del $5^{\circ}$ grado del nivel primario de la Institución Educativa Particular Adventista "Portales del Saber" en Ate-Vitarte. Ha: La aplicación del programa "The pleasure of reading with pictures" tiene efectividad significativa en el desarrollo de la compresión de textos en inglés en el nivel inferencial en los estudiantes del $5^{\circ}$ grado 
del nivel primario de la Institución Educativa Particular Adventista "Portales del Saber” en Ate-Vitarte. Se aplicó el estadístico de prueba $\mathrm{T}$ de student por tratarse de datos paramétricos como ya se explicó previamente.

Tabla 7

T de Student para la dimensión comprensión en nivel inferencial

Diferencias emparejadas

\begin{tabular}{|c|c|c|c|c|c|c|c|}
\hline \multirow[b]{2}{*}{$\underline{\text { Media }}$} & \multirow{2}{*}{$\begin{array}{l}\text { Desviación } \\
\text { estándar }\end{array}$} & \multirow{2}{*}{$\begin{array}{l}\text { Media de } \\
\text { error } \\
\text { estándar }\end{array}$} & \multicolumn{2}{|c|}{$\begin{array}{c}95 \% \text { de intervalo de } \\
\text { confianza de la diferencia } \\
\end{array}$} & \multirow[b]{2}{*}{$\underline{\mathrm{t}}$} & \multirow[b]{2}{*}{$\mathrm{gl}$} & \multirow{2}{*}{$\begin{array}{l}\text { Sig. } \\
\text { (bilateral) }\end{array}$} \\
\hline & & & $\underline{\text { Inferior }}$ & $\underline{\text { Superior }}$ & & & \\
\hline$-6,632$ & 1,012 & ,232 & $-7,119$ & $-6,144$ & $-28,574$ & 18 & ,000 \\
\hline
\end{tabular}

Por los datos obtenidos en la tabla 7 observamos que $\boldsymbol{p}<\mathbf{0 , 0 5}$ (Significancia bilateral,000) por ello podemos afirmar que existe evidencia suficiente para rechazar la hipótesis nula según la regla de decisión; por lo que se acepta la hipótesis alterna: La aplicación del programa "The pleasure of reading with pictures" tiene efectividad significativa en el desarrollo de la comprensión de textos en inglés en nivel inferencial en los estudiantes del 5to. grado de nivel primario de la Institución Educativa Adventista "Portales del Saber" en Ate-Vitarte.

\section{Conclusiones}

El nivel de logro de aprendizaje para el desarrollo de las capacidades de comprensión de textos en inglés del nivel primario de la Institución Educativa Adventista "Portales del Saber" mejoró significativamente, debido a que en el pre test el 63.2\% de los estudiantes se encontraba en el nivel deficiente y el $36.8 \%$ en el nivel regular. En el pos test, superaron en su totalidad al nivel "Bueno". El nivel de logro de aprendizaje para el desarrollo de comprensión de textos en inglés en el nivel literal en los estudiantes del $5^{\circ}$ grado de primaria de la Institución Educativa "Portales del Saber" mejoró en gran manera, debido a que en el pre test el $57.9 \%$ de los estudiantes se encontraba en el nivel deficiente y un $42.1 \%$ en el nivel regular. En el pos test, todos los estudiantes $(100.0 \%)$ alcanzaron al nivel "Bueno". El nivel de logro de aprendizaje para el desarrollo de comprensión de textos en inglés en el nivel inferencial en los estudiantes del $5^{\circ}$ grado de primaria de la Institución Educativa "Portales del Saber" mejoró significativamente debido a que en el pre test el $78.9 \%$ de los estudiantes se encontraba en el nivel deficiente y un $21.1 \%$ en el nivel regular. En el pos test, todos los estudiantes (100.0\%) alcanzaron al nivel "Bueno". Por todo lo antes mencionado, podemos concluir que existe clara evidencia que la aplicación del programa "The pleasure of reading with pictures" a través de los textos iconográficos tuvo relevancia significativa en los estudiantes del $5^{\circ}$ grado de primaria de la Institución Educativa "Portales del Saber".

\section{Recomendaciones}

Considerando que trabajar en matemáticas bajo un enfoque de resolución de problemas aditivos de enunciado verbal, permite un mejor desempeño para un aprendizaje integral del estudiante, blanco prioritario que debe establecer cada docente e institución educativa que brinda un servicio para el desarrollo integral en el estudiante; se dan a conocer las siguientes recomendaciones:

- Aplicar y difundir el programa para otros centros de estudios: instituciones particulares, pero en especial a nuestras instituciones educativas adventistas para mejorar la comprensión de textos en inglés desde el nivel primario, adaptando y mejorando sus lecturas a través de textos iconográficos.

- Realizar el programa con otras muestras, como estudiantes de instituciones educativas estatales donde el nivel de inglés es mínimo para verificar los resultados en otros contextos.

- Adquirir en nuestras Instituciones Educativos Adventistas, un plan lector para cada bimestre que contenga imágenes que aporten al incremento del vocabulario de inglés dentro de lecturas y de esta manera, comprender los textos de una segunda lengua con mayor facilidad. Y si es posible, que el contenido del plan lector sea Cristo céntrico.

- Capacitar a los docentes del área de inglés para que adapten las diferentes lecturas que contienen sus libros de inglés con esta estrategia didáctica: el uso de los textos iconográficos para la mejor comprensión de lo que leen.

- Realizar otras investigaciones más específicas sobre la importancia de las imágenes y actividades lúdicas para el incremento de su vocabulario y la mejor comprensión de textos de una segunda lengua, en este caso el inglés.

- Realizar otras investigaciones en el nivel primario donde se evalúe el desarrollo de la comprensión de textos en inglés en sus tres dimensiones: nivel literal, nivel inferencial y nivel crítico. 


\section{Referencias}

Accotina P., \& Pérez A. (2011). Efectos del programa de estrategias basadas en la activación de conocimientos esquemáticos socioculturales en la comprensión de textos en inglés. (Tesis de Licenciatura). Universidad Nacional de Educación Enrique Guzmán y Valle, Lima, Perú.

Anderson, J. (2001) Aprendizaje y Memoria. México: McGraw Hill.

Ardila, R. (2008). Walden dos. 60 años después. Revista Latinoamericana de Psicología 2008, volumen 40, № 3, $595-597$. Recuperado de: http://www.redalyc.org/pdf/805/80511493013.pdf

Arrieta, B., Batista, J., Meza, R. \& Meza, D. (2006). La comprensión lectora y la redacción académica como centro del curriculum. Acción Pedagógica I C A, No 15 / Enero - diciembre, 2006 - pp. 94-98. Ausubel, D. (1970) Aprendizaje y Cognición. Editorial Euned

Ávila Q.,Tinoco T., \& Valencia U. (2011). Las estrategias del aprendizaje del vocabulario inglés y su relación con la comprensión de textos en inglés. (Tesis de Licenciatura). Universidad Nacional de Educación Enrique Guzmán y Valle, Lima, Perú.

Bestard, J. \& Pérez, M. (1992). La didáctica de la lengua inglesa: Fundamentos Lingüísticos y metodológicos. Madrid: Síntesis

Bulnes, M. (2000) Análisis iconográfico de los textos para la educación bilingüe intercultural. Lima: San

Calero, M. (1998). Educar jugando. Lima: San Marcos

Castillo, L. (2015). Efectos de la programación neurolingüística en el aprendizaje de la asignatura taller de investigación en los estudiantes de inglés-francés. (Tesis doctoral). Universidad Nacional Federico Villareal, Lima, Perú.

Cerrillo, P. \& Yubero, S. (2007). La formación de mediadores para la formación de la Lectura. Cuenca: Artes Plàsticas.Trisogar.

Chumasero O. \& León H. (2012). Los medios audiovisuales y su relación en la comprensión de textos escritos en inglés. (Tesis de Licenciatura). Universidad Nacional de Educación Enrique Guzmán y Valle, Lima, Perú.

Cubas, A. (2007). Actitudes hacia la lectura y niveles de comprensión lectora en estudiantes de sexto grado de primaria (Tesis de Licenciatura en Psicología Educativa). Universidad Católica del Perú. Recuperado de: http://tesis.pucp.edu.pe/repositorio/bitstream/handle/123456789/427/CUBAS_BARRUETO_ANA_ACTITUDES_HAC IA_LECTUR A.pdf?sequence=1

Díaz, F. \& Hernández, G. (2002) Estrategias docentes para un aprendizaje significativo. Una interpretación constructivista (2da. Edición) México: Mc Graw Hill.

Diccionario de la Real Academia (RAE, 2015)

Diccionario etimológico (2016). Recuperado de: http://etimologias.dechile.net/?iconografia

Diseño Curricular Nacional (DCN, 2009). Educación Básica Regular. Proceso de Articulación. Lima: Fimart S.A.C.

Dondis, D. (1973). La sintaxis de la imagen. Barcelona: Gustavo Gill.

Egaña, E. (2004). Oppidum: Imágenes pese a todo. (Memoria visual del holocausto). Recuperado de: http://oppidum.es/2005/PDFs/imagenespeseatodo.pdf

Ferradini, S., \& Tedesco, R. (1997). Lectura de la imagen. Andalucía, España: Red AL y C. Recuperado de:http://www.iar.unicamp.br/lab/luz/ld/Linguagem\%20Visual/lectura_de_la_imagen.pdffile

Fumero, F. (2009). Estrategias Didácticas para la Comprensión de Textos: Una propuesta de investigación acción participativa en el aula. Investigación y postgrado 24 (1). Recuperado de http://www.redalyc.org/pdf/658/65815763003.pdf

García de Díaz, M. (2000). Comprensión Lectora: Niveles y Tipos. (Tesis de Maestría, Universidad Nacional Experimental Francisco de Miranda) Recuperado de: http://www.authorstream.com/Presentation/miriangarciadediaz-1696902comprensi-lectora-tipos-delectura/

Govea, L. (2011). Influencia de la Lectura Extensiva en la Actitud y Comprensión Crítica de Estudiantes de Inglés como Lengua Extranjera. Docencia Universitaria, 12 (2). SADPRO - UCV Universidad Central de Venezuela.

Grabe, W. (1988). "Reassessing the term interactive ". Interactive Approaches to Second Language Reading. Patricia Carrell et al eds. Cambridge: CUP

Grabe, W. (1991). Segundas lenguas. Editorial Ariel. Recuperado de: https://books.google.com.pe/books?id=1 mZiAAAAMAAJ\&q=Grabe+1991+comprension+lectora\&dq=Grabe+1991+co mprension+l ectora\&hl=es-419\&sa=X\&redir_esc $=\mathrm{y}$

Gutiérrez, C. \& Salmerón H. (2012). Estrategias de comprensión lectora: enseñanza y evaluación de la educación primaria. Profesorado. 183202

Harmer, J. (2010). The Practice of English Language Teaching. England: Pearson-Longman.

Hernández, P. \& Maquillón, S. J. (2011). Influencia de la motivación en el rendimiento académico de los estudiantes de formación profesional. Revista Electrónica Interuniversitaria de Formación del Profesorado, Sin mes, 81-100. Recuperado de: http://www.redalyc.org/pdf/2170/217017192007.pdf

Hernández, R., Fernández, C. \& Baptista P. (2010). Metodología de la Investigación. Madrid: Editorial Progreso S.A.

Hogan, P. (2004). Pruebas estadísticas. México: Manual Moderno

Huarcaya, I. \& Crisóstomo, H. (2014). Gestionando los aprendizajes en la escuela. Lima: Ediciones Edicep

Hudson, R. (2011). The cognitive Development of Reading and reading comprenhension. New York: Routledge, Taylor and Francis Group. 
Jiménez, J. (2013) Desarrollo de la Comprensión lectora de Textos multimediales en una lengua extranjera Mediante la enseñanza de Estrategias de lectura: Tesis doctoral, Universidad de Antioquía. Colombia Recuperado de: http://ayura.udea.edu.co:8080/jspui/bitstream/123456789/28/1/PC0756.pdf

Llorente, C. (2000). Imágenes en la enseñanza. Revista de Psicodidáctica. Recuperado de: http://www.redalyc.org/pdf/175/17500911.pdf

López, J., Jiménez, L \& Soto, L. (2009): Iconografía en el proceso de enseñanza lectora del primer año básico en Chile. Recuperado de: http://seminariografica.uchilefau.cl/?p=1206

Marco Común Europea de referencia para las Lenguas (MCER, 2002). Madrid: General de Información y Publicaciones, y Grupo ANAYA, S.A

Méndez, M. (1997). Dimensiones asociadas con el papel de la imagen en material didáctico. Perfiles Educativos, enero-marzo. Recuperado de: http://www.redalyc.org/pdf/132/13207506.pdf

Mendoza, C. (2002). La seducción de las Lecturas en Edades tempranas. Lima: Ministerio de Educación, Cultura y Deporte.

Mendoza, H. (2003). La promoción de la lectura en inglés y su valoración por parte de los alumnos de novela grado de la U.E. "Jacinto Regino Pachano" ubicada en Tocuyo de la Costa, Edo. Falcón. (Tesis para obtener el grado de maestría). Venezuela. Recuperado de: http://es.scribd.com/doc/13501113/Promocion-de-la-Lectura-en-Ingles-Tesis-de-MaestriaPromoting-Reading-in-Elglish-in-the-ESLClassroom\#scribd

MINEDU. (2015). Rutas del Aprendizaje. ¿Qué y cómo aprenden nuestros estudiantes? V ciclo. Área Curricular: Comunicación. Lima: Amauta Impresiones Comerciales S.A.C.

Ministerio de Educación, Cultura y Deporte (1998). Recuperado de: http://www.rtve.es/temas/ministerio-de-educacion-culturaydeporte/14990/12/

Molina, V. (2006). Lectura y educación: los hábitos lectores y su repercusión académica en la Educación Secundaria Obligatoria. Ocnos: Revista de Estudios sobre Lectura, Sin mes, 103-121. Recuperado de https://www.revista.uclm.es/index.php/ocnos/article/view/ocnos_2006.02.07

Montoya, V. (2003). Literatura Infantil. Lenguaje y fantasía. Recuperado de

https://books.google.com.pe/books?id=DCAEGomg8ooC\&pg=PA83\&dq=importancia+de+las+imagenes+en+los+textos $+\mathrm{de}+\mathrm{ni} \% \mathrm{C} 3$

$\% \mathrm{~B} 1$ os\&hl=es\&sa=X\&redir_esc=y\#v=onepage \&q=importancia $\% 20 \mathrm{de} \% 201 \mathrm{las} \% 20 \mathrm{imagenes} \% 20 \mathrm{en} \% 201 \mathrm{los} \% 20$ textos $\% 2$ 0de $\% 20$ ni\% C3\%B1os\&f=false2003)=

Morán C. (1991). El desarrollo de la percepción visual en el aprendizaje de la lectoescritura del alumno del primar grado de la Escuela No 1226 de Vista Alegre U.S.E. no. 14 de Vitarte. (Tesis de Licenciatura). Universidad Peruana Unión, Lima, Perú.

Nunan, D. (1992). Research methods in language learning USA, New York. Cambridge University Press

Nunan, D. (1999). Second Language Teaching learning. Boston-Masachussets. Heinle and Heinly Publishers.

Oñate, T.(2013). Filosofía y Literatura.Madrid: Manuscritos. Recuperado de: https://books.google.com.pe/books?id=b8vQrPzbPCOC\&pg=PT120\&dq=o\%C3\%B1ate $+2013+$ la + lectura \&hl=es$419 \& s a=X \&$ redir_esc $=y \# v=$ onepage $\& q=0 \% C 3 \%$ B1ate $\% 202013 \% 20$ la $\% 2$ 20lectura $\& f=$ false

Ortegano, R. \& Bracamonte, M. (2011). Actividades lúdicas como estrategia didáctica para el mejoramiento de las competencias operacionales en E-a De las matemáticas básicas. (Tesis de Maestría). Universidad de los Andes, Trujillo, Perú. Recuperado de http://tesis.ula.ve/pregrado/tde_arquivos/26/TDE-2012-09-27T06:02:10Z1802/Publico/orteganoramon.pdf

OTP. (2006). Orientaciones para el trabajo pedagógico en el área del Idioma Extranjero

Oxford, R. (1990). Language Learning Strategies. What every teacher should know. Boston: Heinle \& Heinle Publishers

Parodi, G. (2005). Comprensión de textos escritos. 120 pp. Buenos Aires: Eudeba. ISBN: 950-23-1430-1. 10.4067/S071809342006000300009

Pérez, H. (2004). Comprensión y Producción de Textos. Bogotá: Magisterio.

PISA. (2001). Programa Internacional de Evaluación de Estudiantes de la OCDE. Informe Español. Madrid

PISA. (2009). Programa para la Evaluación Internacional de los Alumnos. OCDE. Informe Español. Madrid

PISA. (2009). Reading StatPlanet.PNG. Recuperado de: http://www.statsilk.com/maps/statplanet-world-bank-open-data

Quispe, R. (2015). Relación que existe entre las estrategias metacognitivas de lectura y la comprensión de textos escritos. (Tesis de licenciatura), Universidad Peruana Unión, Lima, Perú.

Rimari, W. (2008). Módulo I: ¿Qué es y cómo desarrollar la comprensión lectora? Revista Innovando. Perú. Número. 20 Rosas, R. \& Sebastián, Ch. (2001) Constructivismo a tres voces. Buenos Aires, Aiqué.

Salazar, S., \& Ponce, D. (1999). Hábitos de Lectura. Lima: Mac Graw Hill

Sánchez, G. (2010). Las estrategias de aprendizaje a través del componente lúdico. Suplementos Marco. ELE. ISSN 1885-2211 / núm. 11, 2010. Recuperado de: http://marcoele.com/descargas/11/sanchez-estrategias-ludico.pdf

Segura Peña, C., Girón Calderón, D., \& Rivera Melgarejo, Y. (1991). El background knowledge y su relación con la comprensión lectora en el área de inglés. (Tesis de Licenciatura). Universidad Nacional de Educación Enrique Guzmán y Valle, Lima, Perú.

Singh \& Singh (1996). Elements of Survey Sampling. Springer-Science Business Media, B.V. doi:10.1007/978-94-017-1404-4

Sole, I. (1992). De la lectura al aprendizaje. Barcelona: Graò. Recuperado de: http://es.slideshare.net/bibliasagaradagloria/de-laimportanciade-la-lectura-isabel 
Solé, I. (2001). Estrategia de Lectura. Barcelona: Graò. Recuperado de :

http://media.utp.edu.co/referenciasbibliograficas/uploads/referencias/libro/1142-estrategias-de-lecturapdf-N0aU6libro.pdf

Torres, A. (2012) Eficacia del programa de comprensión lectora para alumnos de segundo de secundaria en una institución educativa de Ventanilla. (Tesis para optar el grado académico de Maestro en Educación Mención en Aprendizaje y Desarrollo Humano). Recuperado de: http://repositorio.usil.edu.pe/wp-content/uploads/2014/07/2012_Torres_Eficaciadel-programa-decomprensi\%C3\%B3n-lectora-para-alumnos-de-segundo-grado-de-secundaria-en-unainstituci\%C3\%B3n-educativa-de-Ventanilla.pdf

Vera, G. (2013). El pictograma como recurso pedagógico para el desarrollo de habilidades lectoras en niños y niñas del nivel inicial del centro educación básica "Virginia Reyes González” de la Parroquia Anconcito. Cantón Salinas. Provincia de Santa Elena. Ecuador. Recuperado de:

http://repositorio.upse.edu.ec/bitstream/46000/1025/1/TESIS\%20GILMA\%20JUNIO_22\%20EMPASTADA.pdf

Viera, T. (2003). El aprendizaje verbal significativo de Ausubel. Algunas consideraciones desde el enfoque histórico cultural. Recuperado de: <http://www.redalyc.org/articulo.oa?id=37302605> ISSN 0041-8935

White, E. G. (1964). Joyas de los Testimonios. Buenos Aires: Asociación Casa Editora Sudamericana

Zhao, J. (2005). Research in Technology and Second Language Education. Estados Unidos. Recuperado de https://books.google.com.pe/books?id=1RiZEOqHD-

UC\&pg=PA106\&dq=SILBERSTEIN+1987+reading\&hl=en\&sa=X\&ved=0ahUKEwi43e_Kmf_KAhXKth4KHYibAjk

Q6wEIGzAA \#v=onepage\&q=SILBERSTEIN\%201987\%20reading\&f=false

Zorrilla, S. (1993). Introducción a la metodología de la investigación. México, Aguilar León y Cal, Editores, $11^{\text {a }}$ Ed 